\title{
Fuzzy Finite-Time Stability of Chaotic Systems with Time-Varying Delay and Parameter Uncertainties
}

\author{
Dong $\mathrm{Li}^{1,2}$ and Jinde $\mathrm{Cao}^{3,4}$ \\ ${ }^{1}$ School of Automation, Southeast University, Nanjing 210096, China \\ ${ }^{2}$ School of Mathematical Sciences, Anhui University, Hefei 230601, China \\ ${ }^{3}$ Department of Mathematics, Southeast University, Nanjing 210096, China \\ ${ }^{4}$ Department of Mathematics, Faculty of Science, King Abdulaziz University, Jeddah 21589, Saudi Arabia \\ Correspondence should be addressed to Jinde Cao; jdcao@seu.edu.cn
}

Received 27 February 2015; Revised 12 May 2015; Accepted 13 May 2015

Academic Editor: Mohammed Chadli

Copyright (C) 2015 D. Li and J. Cao. This is an open access article distributed under the Creative Commons Attribution License, which permits unrestricted use, distribution, and reproduction in any medium, provided the original work is properly cited.

\begin{abstract}
This paper discusses the finite-time stability of chaotic systems with time-varying delay and parameter uncertainties. A new model based on Takagi-Sugeno (T-S) model is proposed for representing chaotic systems. By the new model, finite-time stability of chaotic systems can be converted into stabilization of fuzzy T-S systems with parameter uncertainties. A sufficient condition is given in terms of matrix inequalities, which guarantees the finite-time stability for fuzzy systems can be achieved. Numerical simulations on the chaotic systems are presented to demonstrate the effectiveness of the theoretical results.
\end{abstract}

\section{Introduction}

In the last decades, the study of controlling chaotic systems has received considerable attention due to its broad applications in biological systems, information processing, secure communications, and so forth. In practical physical systems, the parameters of chaotic systems may not be known exactly. So, there are many papers concerning uncertain chaotic systems $[1,2]$. For the character of the uncertain chaotic systems, many significant results have been obtained by using adaptive control technique, for example, output feedback control [3], fuzzy adaptive control [4], and optimal control [5].

In order to simplify the controller design of the chaotic systems, various schemes have been developed, among which fuzzy model is one of the successful approaches to obtain nonlinear chaotic systems. Specifically, a so-called TakagiSugeno (TS) fuzzy model was proposed in [6], where complex nonlinear systems are represented by some local linear subsystems. Based on the T-S fuzzy model, some chaotic control design methods have been developed [7-12]. In [7], a fuzzy model is presented to simulate two different chaotic systems with different numbers of nonlinear terms and a new adaptive approach is proposed to synchronize these two different fuzzy chaotic systems. Predictive control for chaotic systems is based on a T-S fuzzy model in [8]. Wang and $\mathrm{Wu}$ [9] and Zhang et al. [10] investigate the problem of fuzzy impulsive control to stabilize chaotic systems.

Although there have been many works to discuss the stabilization of chaotic systems, most of the existing stabilization algorithms are asymptotically convergent algorithms [13-17], which means that the convergence rate is at best exponential with infinite settling time. Compared to the asymptotically convergent algorithms, the finite-time convergence algorithms demonstrate not only faster convergence rates, but also better disturbance rejection properties and robustness against uncertainties [18-20]. References [21-24] discuss finite-time control for chaotic systems. But there are few works considering finite-time control for chaotic systems based on the fuzzy models.

Motivated by the aforementioned analysis, the finite-time stabilization control for chaotic systems based on the T-S fuzzy models is a significative topic. Our main contribution in this paper is the introduction of the fuzzy model to discuss finite-time stabilization of chaotic systems in a general model. In this model, we not only consider time-varying delay, but 
also study the chaotic system with parameter uncertainties. Obviously, on the one hand, our results have optimality in the convergence time of stabilization in $[14,15]$; on the other hand, they are more practically applicable than those in [22-24]. Based on the Lyapunov stability theory, a feedback controller is designed to guarantee the stabilization in finite time. The example is given to illustrate our theoretical results.

The rest of the paper is organized as follows. In Section 2, some preliminaries are briefly given. Section 3 presents the main results. In Section 4, simulation results aiming at substantiating the theoretical analysis are presented. This paper is concluded in Section 5.

\section{Preliminaries and Problem Formulation}

In this paper, we want to develop a finite-time control law for a class of chaotic systems. In system analysis and design, it is important to select an appropriate model representing a real system. The T-S fuzzy model can express a highly nonlinear functional relation with a small number of rules. Many chaotic systems can be represented by fuzzy linear models, such as Lorenz, Rössler, Chua, Chen, and Lü systems. This fuzzy modeling method is simple and natural. So, consider a class of time-delayed chaotic systems represented by the following time-delayed T-S fuzzy model.

Plant Rule is as follows.

$$
\begin{aligned}
& \text { If } \theta_{1}(t) \text { is } M_{i 1}, \ldots \text {, and } \theta_{p}(t) \text { is } M_{i p} \text {, then } \\
& \dot{x}(t)=A_{i} x(t)+B_{i} x(t-\tau(t)), \quad i=1,2, \ldots, N \text {, }
\end{aligned}
$$

where $x(t)=\left(x_{1}(t), \ldots, x_{n}(t)\right)^{T} \in R^{n}$ is the state variable; the premise variables $\theta_{1}(t), \ldots, \theta_{p}(t)$ are proper state variables; $N$ is the number of the fuzzy rules; $M_{i j}(j=1,2, \ldots, p)$ are fuzzy sets; $A_{i}$ and $B_{i}$ are known constant matrices with appropriate dimensions; $\tau(t)$ is the transmission delay.

In practice, however, some parameters of chaotic systems cannot be exactly known a priori. If the information of uncertain matrices is considered, the results will be less conservative than those results that do not utilize the elemental uncertain information. Thus, it is very interesting to study the design of the fuzzy systems with parameter perturbations.

Plant Rule is as follows.

$$
\begin{aligned}
& \text { If } \theta_{1}(t) \text { is } M_{i 1}, \ldots \text {, and } \theta_{p}(t) \text { is } M_{i p} \text {, then } \\
& \begin{aligned}
\dot{x}(t)=\left(A_{i}+\nabla A_{i}\right) x(t)+\left(B_{i}+\nabla B_{i}\right) x( & t-\tau(t)), \\
& i=1,2, \ldots, N .
\end{aligned}
\end{aligned}
$$

The parameter uncertainties are classically written as [10] $\nabla A_{i}=D_{i}^{A} F_{i}^{A} E_{i}^{A}$ and $\nabla B_{i}=D_{i}^{B} F_{i}^{B} E_{i}^{B}$ where $D_{i}^{A}, D_{i}^{B}, E_{i}^{A}, E_{i}^{B}$ are known real matrices of appropriate dimension and $F_{i}^{A}, F_{i}^{B}$ are the unknown matrix functions with Lebesgue-measurable elements and satisfy the conditions $F_{i}^{A^{T}} F_{i}^{A} \leq I, F_{i}^{B^{T}} F_{i}^{B} \leq I$, where $I$ is the identity matrix of appropriate dimension.
The overall fuzzy system with control is inferred as follows:

$$
\begin{gathered}
\dot{x}(t)=\sum_{i=1}^{N} h_{i}(\theta(t))\left[\left(A_{i}+\nabla A_{i}\right) x(t)\right. \\
\left.+\left(B_{i}+\nabla B_{i}\right) x(t-\tau(t))+u(t)\right],
\end{gathered}
$$

where $u(t)$ denotes the feedback control, $h_{i}(\theta(t))=\omega_{i}(\theta(t)) /$ $\sum_{i=1}^{N} \omega_{i}(\theta(t)), \omega_{i}(\theta(t))=\prod_{j=1}^{p} M_{i j}\left(\theta_{j}(t)\right)$, and $\omega_{i}(\theta(t)) \geq 0$, $\sum_{i=1}^{N} \omega_{i}(\theta(t)) \geq 0$; thus $h_{i}(\theta(t)) \geq 0, \sum_{i=1}^{N} h_{i}(\theta(t))=1$.

Assume $C\left([-\tau, 0], R^{n}\right)$ is a Banach space of continuous functions mapping the interval $[-\tau, 0]$ into $R^{n}$ with the norm $\|\phi\|=\sup _{-\tau \leq \theta \leq 0}\|\phi(\theta)\|$. For the functional differential equation (3), its initial conditions are given by $x_{i}(t)=$ $\phi_{i}(t) \in C\left([-\tau, 0], R^{n}\right)$. We always assume that (3) has a unique solution with respect to initial conditions.

For starting simplification, one has the following fundamental assumption.

Assumption 1. Consider $0 \leq \dot{\tau}(t) \leq h<1$, where $h$ is constant.

To end this section, we introduce the following lemmas which are useful in deriving sufficient conditions of finitetime stability.

Lemma 2 (see [25]). Consider system $\dot{x}=f(x), f(0)=0$, $x \in R^{n}$, where $f(\cdot): R^{n} \rightarrow R^{n}$ is a continuous vector function. Suppose there exist a $C^{1}$ positive definite and proper function $V: R^{n} \rightarrow R$ and real numbers $\mu>0$ and $\eta \in(0,1)$ such that $\dot{V}+\mu V^{\eta}$ is negative semidefinite. Then the origin is a globally finite-time stable equilibrium of system $\dot{x}=f(x)$. Moreover, the settling time $T \leq V^{1-\eta}(0) /(1-\eta) \mu$.

Lemma 3 (see [26]). Given any real matrices $A, B, \Sigma$ of appropriate dimensions and a scalar $s>0$, such that $0<\Sigma=$ $\Sigma^{T}$, then the following inequality holds:

$$
A^{T} B+B^{T} A \leq s A^{T} \sum A+s^{-1} B^{T} \Sigma^{-1} B .
$$

Lemma 4 (see [27]). Let $A, D, E$, and $F$ be real matrices of appropriate dimensions, with $F$ satisfying $\|F\| \leq 1$. Then one has the following.

(a) For any scalar $\lambda>0$,

$$
D F E+E^{T} F^{T} D^{T} \leq \lambda^{-1} D D^{T}+\lambda E E^{T} .
$$

(b) For any matrix $P>0$ and scalar $\xi>0$ such that $\xi I-$ $E^{T} P E>0$,

$$
\begin{aligned}
& (A+D F E)^{T} P(A+D F E) \\
& \quad \leq A^{T} P A+A^{T} P E\left(\xi I-E^{T} P E\right)^{-1} E^{T} P A+\xi^{-1} D^{T} D .
\end{aligned}
$$

Lemma 5 ((Schur complement) [28]). For a given matrix

$$
S=\left[\begin{array}{ll}
S_{11} & S_{12} \\
S_{12}^{T} & S_{22}
\end{array}\right]<0,
$$

where $S_{11}=S_{11}^{T}$ and $S_{22}=S_{22}^{T}$ are equivalent to any one of the following conditions: 
(a) $S_{22}<0, S_{11}-S_{12} S_{22}^{-1} S_{12}^{T}<0$;

(b) $S_{11}<0, S_{22}-S_{12}^{T} S_{11}^{-1} S_{12}<0$.

\section{Finite-Time Stabilization}

First, we present our main result on the finite-time stabilization of system (3).

Theorem 6. Suppose the positive constants $\lambda_{i}, i=1,2,3, k_{1}$, $k_{2}$, and positive definite matrix $P$, such that

(1)

$$
\begin{gathered}
\left(\begin{array}{ccc}
A_{i}^{T} P+P A_{i}+\left(\lambda_{2}+k_{2}-k_{1}\right) P & E_{i}^{A} & P D_{i}^{A} \\
E_{i}^{A^{T}} & -\lambda_{1} I & 0 \\
D_{i}^{A^{T}} P & 0 & -\lambda_{1}^{-1} I
\end{array}\right) \\
\leq 0, \quad i=1, \ldots, N,
\end{gathered}
$$

(2)

$$
\begin{aligned}
& \left(\begin{array}{ccc}
B_{i}^{T} P B_{i}-k_{2} \lambda_{2}(1-h) P & D_{i}^{B} & E_{i}^{B^{T}} P B_{i} \\
D_{i}^{B^{T}} & -\lambda_{3}^{-1} I & 0 \\
B_{i}^{T} P E_{i}^{B} & 0 & -\left(\lambda_{3} I-E_{i}^{B^{T}} P E_{i}^{B}\right)
\end{array}\right) \\
& \leq 0, \quad i=1, \ldots, N .
\end{aligned}
$$

Then, stabilization of system (3) under Assumption 1 can be achieved in finite time, if the control law $u$ is designed as

$$
\begin{aligned}
u=- & \frac{k_{1}}{2} x(t)-\frac{\mu}{2} \frac{\lambda_{\max }^{(\eta+1) / 2}(P)}{\lambda_{\min }(P)} \operatorname{sign}(x(t))|x(t)|^{\eta} \\
- & \frac{\mu}{2 \lambda_{\min }(P)}\left(k_{2} \int_{t-\tau}^{t} x^{T}(s) P x(s) \mathrm{d} s\right)^{(1+\eta) / 2} \\
& \cdot \frac{x(t)}{\|x(t)\|^{2}},
\end{aligned}
$$

where $\mu$ is an arbitrary positive constant.

Proof. Construct a Lyapunov function:

$$
V(t)=x^{T}(t) P x(t)+k_{2} \int_{t-\tau}^{t} x^{T}(s) P x(s) \mathrm{d} s,
$$

so, we get

$$
\begin{aligned}
& \left.\dot{V}(t)\right|_{(3)}=\sum_{i=1}^{N} h_{i}(\theta(t))\left\{x^{T}(t)\right. \\
& \cdot\left(A_{i}^{T} P+P A_{i}+E_{i}^{A^{T}} F_{i}^{A^{T}} D_{i}^{A^{T}} P+P D_{i}^{A} F_{i}^{A} E_{i}^{A}\right) x(t) \\
& \quad+x^{T}(t-\tau)\left(B_{i}^{T}+E_{i}^{B^{T}} F_{i}^{B^{T}} D_{i}^{B^{T}}\right) P x(t)+x^{T}(t) \\
& \left.\cdot P\left(B_{i}+D_{i}^{A} F_{i}^{A} E_{i}^{A}\right) x(t-\tau)+2 x^{T}(t) P u(t)\right\} \\
& +k_{2} x^{T}(t) P x(t)-k_{2} x^{T}(t-\tau) P x(t-\tau)(1-\dot{\tau}(t)) .
\end{aligned}
$$

Using Lemma 4(a), we have

$$
\begin{aligned}
& E_{i}^{A^{T}} F_{i}^{A^{T}} D_{i}^{A^{T}} P+P D_{i}^{A} F_{i}^{A} E_{i}^{A} \\
& \quad \leq \lambda_{1}^{-1} P D_{i}^{A} D_{i}^{A^{T}} P+\lambda_{1} E_{i}^{A} E_{i}^{A^{T}} .
\end{aligned}
$$

Applying Lemmas 3 and 4(b), we get

$$
\begin{aligned}
& x^{T}(t-\tau)\left(B_{i}^{T}+E_{i}^{B^{T}} F_{i}^{B^{T}} D_{i}^{B^{T}}\right) P x(t)+x^{T}(t) P\left(B_{i}\right. \\
& \left.+D_{i}^{B} F_{i}^{B} E_{i}^{B}\right) x(t-\tau) \leq \lambda_{2}^{-1} x^{T}(t-\tau)\left(B_{i}^{T}\right. \\
& \left.+E_{i}^{B^{T}} F_{i}^{B^{T}} D_{i}^{B^{T}}\right) P\left(B_{i}+D_{i}^{B} F_{i}^{B} E_{i}^{B}\right) x(t-\tau) \\
& +\lambda_{2} x^{T}(t) P x(t) \leq \lambda_{2}^{-1} x^{T}(t-\tau)\left(B_{i}^{T} P B_{i}\right. \\
& +B_{i}^{T} P E_{i}^{B}\left(\lambda_{3} I-E_{i}^{B^{T}} P E_{i}^{B}\right)^{-1} E_{i}^{B^{T}} P B_{i} \\
& \left.+\lambda_{3}^{-1} D_{i}^{B^{T}} D_{i}^{B}\right) x(t-\tau)+\lambda_{2} x^{T}(t) P x(t)
\end{aligned}
$$

where $\lambda_{i}, i=1,2,3$, are positive constants.

Substituting (13)-(14) into (12) and using Assumption 1, we have

$$
\begin{aligned}
\dot{V}( & t) \leq \sum_{i=1}^{N} h_{i}(\theta(t))\left\{x ^ { T } ( t ) \left(A_{i}^{T} P+P A_{i}\right.\right. \\
& \left.+\lambda_{1}^{-1} P D_{i}^{A} D_{i}^{A^{T}} P+\lambda_{1} E_{i}^{A} E_{i}^{A^{T}}+\lambda_{2} P+k_{2} P\right) x(t) \\
& +2 x^{T}(t) P u(t)+x^{T}(t-\tau)\left[\lambda _ { 2 } ^ { - 1 } \left(B_{i}^{T} P B_{i}\right.\right. \\
& +B_{i}^{T} P E_{i}^{B}\left(\lambda_{3} I-E_{i}^{B^{T}} P E_{i}^{B}\right)^{-1} E_{i}^{B^{T}} P B_{i} \\
& \left.\left.\left.+\lambda_{3}^{-1} D_{i}^{B^{T}} D_{i}^{B}\right)-k_{2} P(1-h)\right] x(t-\tau)\right\} .
\end{aligned}
$$

Applying Schur criterion on conditions (1) and (2) yields

$$
\begin{aligned}
& A_{i}^{T} P+P A_{i}+\lambda_{1}^{-1} P D_{i}^{A} D_{i}^{A^{T}} P+\lambda_{1} E_{i}^{A} E_{i}^{A^{T}}+\lambda_{2} P \\
& \quad+k_{2} P \leq k_{1} P \\
& B_{i}^{T} P B_{i}+B_{i}^{T} P E_{i}^{B}\left(\lambda_{3} I-E_{i}^{B^{T}} P E_{i}^{B}\right)^{-1} E_{i}^{B^{T}} P B_{i} \\
& \quad+\lambda_{3}^{-1} D_{i}^{B^{T}} D_{i}^{B} \leq k_{2} \lambda_{2}(1-h) P .
\end{aligned}
$$


Substituting (16) into (15) and using control inputs in (10), one obtains

$$
\begin{aligned}
\dot{V}(t) & \leq-\mu \frac{\lambda_{\max }^{(1+\eta) / 2}(P)}{\lambda_{\min }(P)} x^{T}(t) P \operatorname{sign}(x(t))|x(t)|^{\eta} \\
& -\frac{\mu}{\lambda_{\min }(P)}\left(k_{2} \int_{t-\tau}^{t} x^{T}(s) P x(s) \mathrm{d} s\right)^{(1+\eta) / 2} \\
& \frac{x(t)^{T} P x(t)}{\|x(t)\|^{2}} \\
& \leq-\mu\left[\lambda_{\max }^{(1+\eta) / 2}(P)\left(x^{T}(t) x(t)\right)^{(1+\eta) / 2}\right. \\
& \left.+\left(k_{2} \int_{t-\tau}^{t} x^{T}(s) P x(s) \mathrm{d} s\right)^{(1+\eta) / 2}\right] \\
& \leq-\mu(V(t))^{(1+\eta) / 2} .
\end{aligned}
$$

Therefore, by Lemma 2 the synchronization error system (3) is globally finite-time stable and the finite time is estimated by

$$
T=t_{0}+\frac{V^{1-(1+\eta) / 2}\left(t_{0}\right)}{\mu(1-(1+\eta) / 2)} .
$$

This completes the proof of the theorem.

Remark 7. In $[10,14,15]$, the authors investigated the stabilization of chaotic systems via fuzzy models. But these results were all based on the stability time in large enough. In Theorem 6, we introduce the feedback control method to guarantee finite-time stability.

Remark 8. Theorem 6 provides a sufficient condition of finite-time stabilization of chaotic systems. In [22-24], the authors also study the finite-time control for chaotic systems, but the delay is a constant or there is no delay; moreover the parameters of chaotic systems are known exactly. Obviously, the results of this section extend and improve existing results.

Remark 9. The magnitude of $x(t) /\|x(t)\|^{2}$ in the controller $u(t)$ will turn to infinity as $x(t) \rightarrow 0$; in order to avoid the occurrence of this phenomenon, we can add a sufficient small positive constant $\varepsilon$ to its denominator in practice $[2,29]$.

Similar to Huang et al. [30] and Khoo et al. [31], the candidate Lyapunov function $V(t)$ can be chosen as $V(t)=$ $x^{T}(t) x(t)$. A summary is shown in the next corollary.

Corollary 10. Suppose the positive constants $\lambda_{i}, i=1,2,3$, $k_{1}, k_{2}$, and positive constant $p$, such that

(1)

$$
\begin{aligned}
A_{i}^{T} p & +p A_{i}+\lambda_{1}^{-1} p^{2} D_{i}^{A} D_{i}^{A^{T}}+\lambda_{1} E_{i}^{A} E_{i}^{A^{T}} \\
& +\left(\lambda_{2}+k_{2}-k_{1}\right) p I \leq 0, \quad i=1, \ldots, N
\end{aligned}
$$

$$
\begin{aligned}
& \lambda_{2}^{-1}\left(p B_{i}^{T} B_{i}+p^{2} B_{i}^{T} E_{i}^{B}\left(\lambda_{3} I-p E_{i}^{B^{T}} E_{i}^{B}\right)^{-1} E_{i}^{B^{T}} B_{i}\right. \\
& \left.\quad+\lambda_{3}^{-1} D_{i}^{B^{T}} D_{i}^{B}\right)-k_{2}(1-h) p I \leq 0, \quad i=1, \ldots, N .
\end{aligned}
$$

Then, stabilization of system (3) under Assumption 1 can be achieved in finite time, if the control law $u$ is designed as

$$
\begin{aligned}
u=- & \frac{k_{1}}{2} x(t)-\frac{\mu}{2} p^{(\eta-1) / 2} \operatorname{sign}(x(t))|x(t)|^{\eta}-\frac{\mu}{2} \\
& \cdot p^{(\eta-1) / 2}\left(k_{2} \int_{t-\tau}^{t} x^{T}(s) x(s) \mathrm{d} s\right)^{(1+\eta) / 2} \\
& \cdot \frac{x(t)}{\|x(t)\|^{2}}
\end{aligned}
$$

where $\mu$ is an arbitrary positive constant.

Proof. Letting $P=p I$, we can obtain Corollary 10 directly from Theorem 6 .

\section{Numerical Example}

In this section, simulation example is presented to illustrate the utility of theoretical analysis in this paper.

Example 1. We consider the following time-delayed Lorenz system [32]:

$$
\begin{aligned}
& \dot{x}_{1}(t)=-10 x_{1}(t)+10 x_{2}(t-\tau), \\
& \dot{x}_{2}(t)=28 x_{1}(t)-x_{2}(t)-x_{1}(t) x_{3}(t), \\
& \dot{x}_{3}(t)=x_{1}(t) x_{2}(t)-\frac{8}{3 x_{3}(t-\tau)},
\end{aligned}
$$

where $\tau=1 / 6$. Figure 1 is the state trajectory of system (22).

Then we have the following fuzzy control model with parameter uncertainties.

Plant Rule is as follows. If $x_{1}(t)$ is $M_{i}$, then

$$
\begin{aligned}
\dot{x}(t)= & \left(A_{i}+\nabla A_{i}\right) x(t)+\left(B_{i}+\nabla B_{i}\right) x(t-\tau(t)) \\
& +u(t), \quad i=1,2,
\end{aligned}
$$




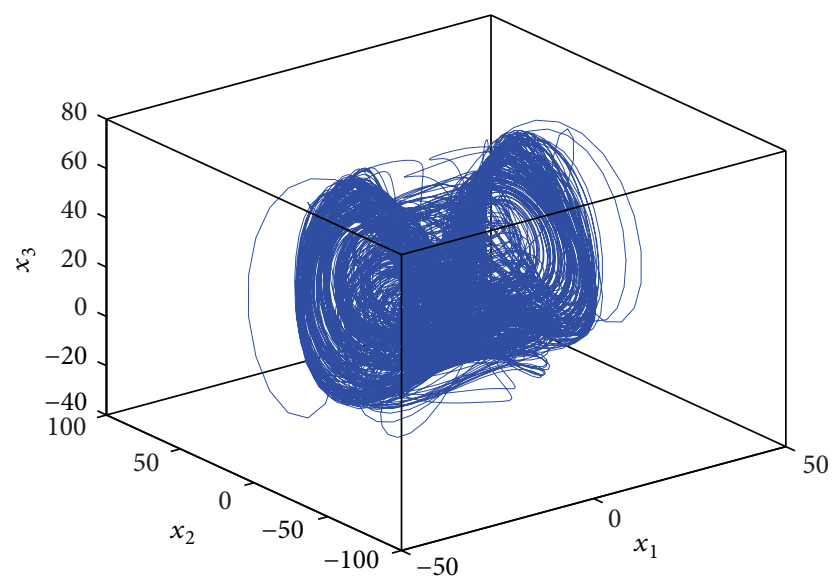

FIGURE 1: The state trajectory of system (22).

where $x(t)=\left(x_{1}(t), x_{2}(t), x_{3}(t)\right)^{T}, M_{1}=(1 / 2)\left(1+x_{1}(t) / 30\right)$, $M_{2}=(1 / 2)\left(1-x_{1}(t) / 30\right)$,

$$
\begin{aligned}
& A_{1}=\left(\begin{array}{ccc}
-10 & 0 & 0 \\
28 & -1 & -30 \\
0 & 30 & 0
\end{array}\right), \\
& A_{2}=\left(\begin{array}{ccc}
-10 & 0 & 0 \\
28 & -1 & 30 \\
0 & -30 & 0
\end{array}\right), \\
& B_{1}=B_{2}=\left(\begin{array}{ccc}
0 & 10 & 0 \\
0 & 0 & 0 \\
0 & 0 & -\frac{8}{3}
\end{array}\right) .
\end{aligned}
$$

The elements of $\nabla A_{i}$ and $\nabla B_{i}$ are randomly chosen within $20 \%$ of their nominal values corresponding to $A_{i}$ and $B_{i}$, respectively. Based on assumption of uncertainty, we define $F_{i}^{A}$ and $F_{i}^{B}$ to be random matrices and satisfy the conditions $F_{i}^{A^{T}} F_{i}^{A} \leq I, F_{i}^{B^{T}} F_{i}^{B} \leq I$,

$$
\begin{aligned}
& D_{i}^{A}=D_{i}^{B}=\operatorname{diag}(0.2,0.2,0.2), \\
& E_{i}^{A}=\left(\begin{array}{ccc}
-10 & 0 & 0 \\
28 & -1 & 0 \\
0 & 0 & 0
\end{array}\right), \\
& E_{i}^{B}=B_{i},
\end{aligned}
$$$$
i=1,2 \text {. }
$$

Select $\lambda_{1}=\lambda_{2}=1, \lambda_{3}=15, k_{1}=100, k_{2}=20$, and $\eta=0.25$. By the simulation of the Matlab LMI Toolbox, $P=$ $[0.1345,-0.2493,0.0000 ;-0.2493,10.2021,-0.0000 ; 0.0000$, $-0.0000,1.3286]$. The trajectory of system states $x_{i}(t)$ under finite-time control is shown in Figure 2. Then the simulation results show the correctness of Theorem 6 .

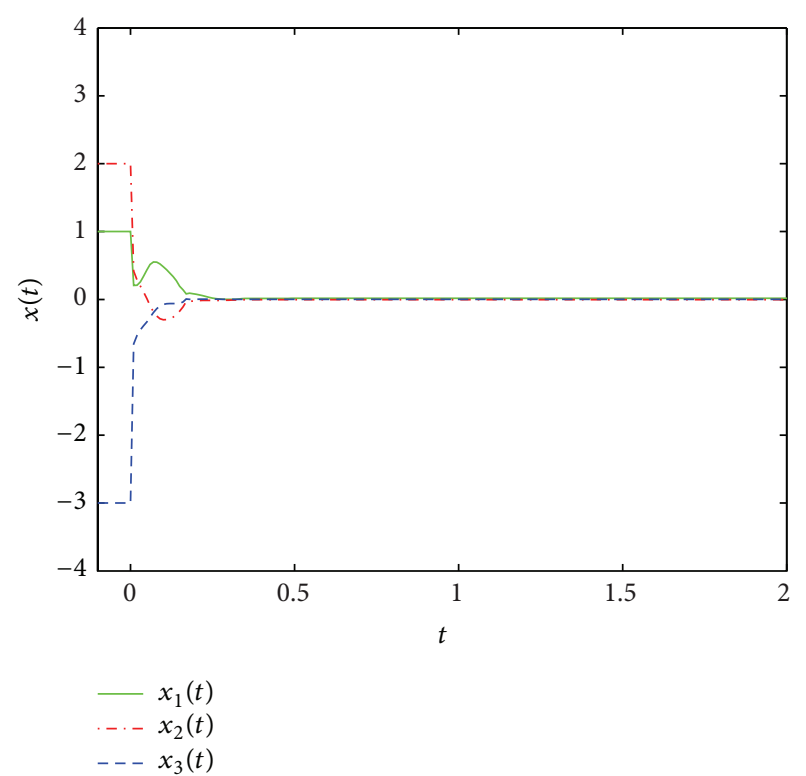

FIGURE 2: The trajectory of system states $x_{i}(t)$ with finite-time control.

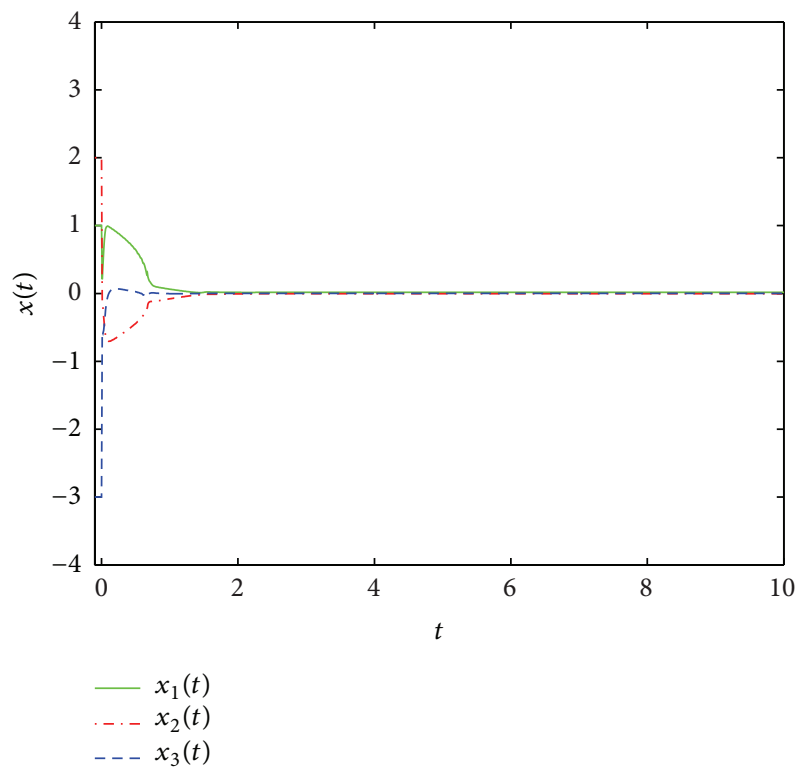

FIGURE 3: The trajectory of system (22) with $\tau(t)=e^{t} /\left(1+e^{t}\right)$ via finite-time control.

More generally, we can choose a time-varying delay to verify the correctness of the theory. We choose the delay as $\tau(t)=e^{t} /\left(1+e^{t}\right)$ in system (22). Obviously, $0<$ $\dot{\tau}(t)<1 / 2<1$. By the simulation of the Matlab LMI Toolbox, $P=[0.1439,-0.3021,0.0004 ;-0.3021,7.8229$, $0.0856 ; 0.0004,0.0856,1.7595]$. The trajectory of system states $x_{i}(t)$ under finite-time control is shown in Figure 3.

Remark 11. When $\tau$ is a time-varying delay, system (22) may not be a chaotic system. In [33], when the systems have no 
chaotic phenomenon, the method is not feasible. However, our results do not need this restriction.

\section{Conclusions}

This paper presents finite-time stability of chaotic systems with time-varying delay by fuzzy-model-based controllers. In order to obtain better robustness results, we discuss the fuzzy system with parameter uncertainties. Various sufficient conditions for finite-time control are derived by Lyapunov methods and linear matrix inequalities (LMI) techniques. These results are novel; some numerical examples are given to verify our theoretical results. Next, we will continue the finitetime control problem for coupled chaotic systems based on the T-S fuzzy models.

\section{Conflict of Interests}

The authors declare that there is no conflict of interests regarding the publication of this paper.

\section{References}

[1] J. Mei, M. Jiang, and J. Wang, "Finite-time structure identification and synchronization of drive-response systems with uncertain parameter," Communications in Nonlinear Science and Numerical Simulation, vol. 18, no. 4, pp. 999-1015, 2013.

[2] M. P. Aghababa and H. P. Aghababa, "A general nonlinear adaptive control scheme for finite-time synchronization of chaotic systems with uncertain parameters and nonlinear inputs," Nonlinear Dynamics, vol. 69, no. 4, pp. 1903-1914, 2012.

[3] J. Zhou and M. J. Er, "Adaptive output control of a class of uncertain chaotic systems," Systems and Control Letters, vol. 56, no. 6, pp. 452-460, 2007.

[4] A. Boulkroune, A. Bouzeriba, S. Hamel, and T. Bouden, "A projective synchronization scheme based on fuzzy adaptive control for unknown multivariable chaotic systems," Nonlinear Dynamics, vol. 78, no. 1, pp. 433-447, 2014.

[5] Y. Miladi, M. Feki, and N. Derbel, "Stabilizing the unstable periodic orbits of a hybrid chaotic system using optimal control," Communications in Nonlinear Science and Numerical Simulation, vol. 20, no. 3, pp. 1043-1056, 2015.

[6] T. Takagi and M. Sugeno, "Fuzzy identification of systems and its applications to modeling and control," IEEE Transactions on Systems, Man and Cybernetics, vol. 15, no. 1, pp. 116-132, 1985.

[7] S.-Y. Li, H.-K. Chen, L.-M. Tam, S.-C. Huang, and Z.-M. Ge, "Pragmatical adaptive synchronization-new fuzzy model of two different and complex chaotic systems by new adaptive control," Information Sciences, vol. 277, pp. 458-480, 2014.

[8] A. Senouci and A. Boukabou, "Predictive control and synchronization of chaotic and hyperchaotic systems based on a T-S fuzzy model," Mathematics and Computers in Simulation, vol. 105, pp. 62-78, 2014.

[9] Z.-P. Wang and H.-N. Wu, "Synchronization of chaotic systems using fuzzy impulsive control," Nonlinear Dynamics, vol. 78, no. 1, pp. 729-742, 2014.

[10] X. Zhang, A. Khadra, D. Yang, and D. Li, "Unified impulsive fuzzy-model-based controllers for chaotic systems with parameter uncertainties via LMI," Communications in Nonlinear
Science and Numerical Simulation, vol. 15, no. 1, pp. 105-114, 2010.

[11] C. K. Ahn, "Fuzzy delayed output feedback synchronization for time-delayed chaotic systems," Nonlinear Analysis: Hybrid Systems, vol. 4, no. 1, pp. 16-24, 2010.

[12] K. Yuan, H.-X. Li, and J. Cao, "Robust stabilization of the distributed parameter system with time delay via fuzzy control," IEEE Transactions on Fuzzy Systems, vol. 16, no. 3, pp. 567-584, 2008.

[13] W. He and J. Cao, "Adaptive synchronization of a class of chaotic neural networks with known or unknown parameters," Physics Letters A, vol. 372, no. 4, pp. 408-416, 2008.

[14] M. H. Asemani and V. J. Majd, "Stability of output-feedback DPDC-based fuzzy synchronization of chaotic systems via LMI," Chaos, Solitons and Fractals, vol. 42, no. 2, pp. 1126-1135, 2009.

[15] M. S. Ali and P. Balasubramaniam, "Global exponential stability of uncertain fuzzy BAM neural networks with time-varying delays," Chaos, Solitons \& Fractals, vol. 42, no. 4, pp. 2191-2199, 2009.

[16] M. Chadli, I. Zelinka, and T. Youssef, "Unknown inputs observer design for fuzzy systems with application to chaotic system reconstruction," Computers \& Mathematics with Applications, vol. 66, no. 2, pp. 147-154, 2013.

[17] M. Chadli and I. Zelinka, "Chaos synchronization of unknown inputs Takagi-Sugeno fuzzy: application to secure communications," Computers and Mathematics with Applications, vol. 68, no. 12, pp. 2142-2147, 2014.

[18] H. Du, S. Li, and C. Qian, "Finite-time attitude tracking control of spacecraft with application to attitude synchronization," IEEE Transactions on Automatic Control, vol. 56, no. 11, pp. 2711-2717, 2011.

[19] S. Ding, S. Li, and Q. Li, "Disturbance analysis for continuous finite-time control systems," Journal of Control Theory and Applications, vol. 7, pp. 271-276, 2009.

[20] S. Li, H. Du, and X. Lin, "Finite-time consensus algorithm for multi-agent systems with double-integrator dynamics," Automatica, vol. 47, no. 8, pp. 1706-1712, 2011.

[21] E. Moulay, M. Dambrine, N. Yeganefar, and W. Perruquetti, "Finite-time stability and stabilization of time-delay systems," Systems \& Control Letters, vol. 57, no. 7, pp. 561-566, 2008.

[22] J. Mei, M. Jiang, W. Xu, and B. Wang, "Finite-time synchronization control of complex dynamical networks with time delay," Communications in Nonlinear Science and Numerical Simulation, vol. 18, no. 9, pp. 2462-2478, 2013.

[23] J. Huang, C. Li, T. Huang, and X. He, "Finite-time lag synchronization of delayed neural networks," Neurocomputing, vol. 139, pp. 145-149, 2014.

[24] Q. Wei, X. Wang, and X. Hu, "Finite-time hybrid projective synchronization of unified chaotic system," Transactions of the Institute of Measurement and Control, vol. 36, no. 8, pp. 10691073, 2014.

[25] S. P. Bhat and D. S. Bernstein, "Geometric homogeneity with applications to finite-time stability," Mathematics of Control, Signals, and Systems, vol. 17, no. 2, pp. 101-127, 2005.

[26] S. Bovd, L. Ghaoui, E. E. I. Feron, and V. Balakrishnan, Linear Matrix Inequalities in System and Control Theory, SIAM, Philadephia, Pa, USA, 1994.

[27] C. E. de Souza and X. Li, "Delay-dependent robust $H_{\infty}$ control of uncertain linear state-delayed systems," Automatica, vol. 35, no. 7, pp. 1313-1321, 1999. 
[28] S. Boyd, L. El Ghaoui, E. Feron, and V. Balakrishnan, Linear Matrix Inequalities in System and Control Theory, vol. 15 of SIAM Studies in Applied Mathematics, SIAM, Philadelphia, Pa, USA, 1994.

[29] L. Zhou, C. Wang, H. He, and Y. Lin, "Time-controllable combinatorial inner synchronization and outer synchronization of anti-star networks and its application in secure communication," Communications in Nonlinear Science and Numerical Simulation, vol. 22, no. 1-3, pp. 623-640, 2015.

[30] X. Huang, W. Lin, and B. Yang, "Global finite-time stabilization of a class of uncertain nonlinear systems," Automatica, vol. 41, no. 5, pp. 881-888, 2005.

[31] S. Khoo, L. Xie, and Z. Man, "Robust finite-time consensus tracking algorithm for multirobot systems," IEEE/ASME Transactions on Mechatronics, vol. 14, no. 2, pp. 219-228, 2009.

[32] L. Li, H. Peng, Y. Yang, and X. Wang, "On the chaotic synchronization of Lorenz systems with time-varying lags," Chaos, Solitons and Fractals, vol. 41, no. 2, pp. 783-794, 2009.

[33] U. E. Vincent and R. Guo, "Finite-time synchronization for a class of chaotic and hyperchaotic systems via adaptive feedback controller," Physics Letters A, vol. 375, no. 24, pp. 2322-2326, 2011. 


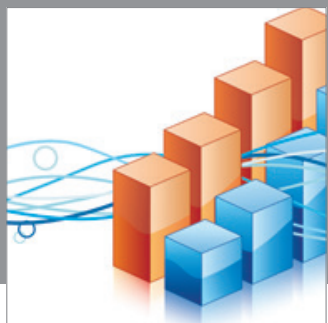

Advances in

Operations Research

mansans

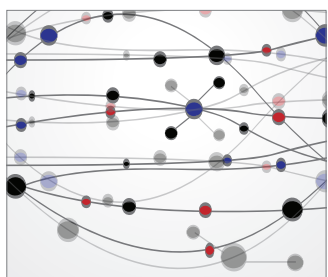

The Scientific World Journal
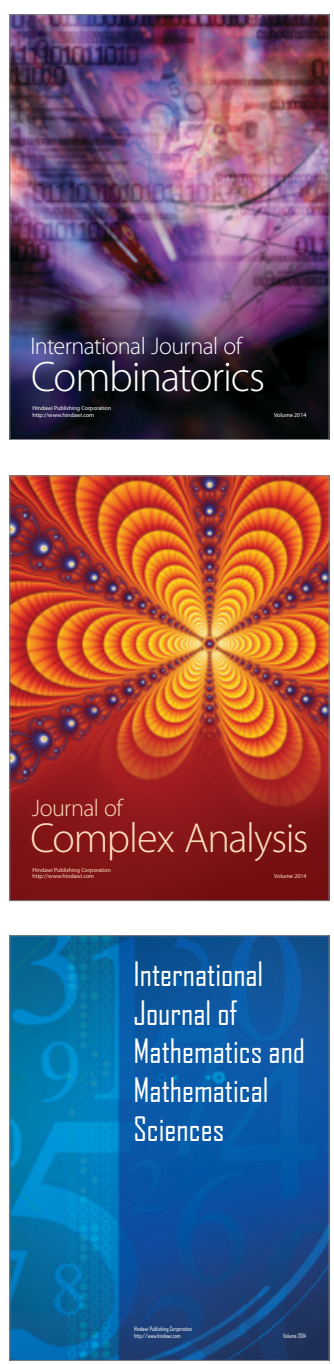
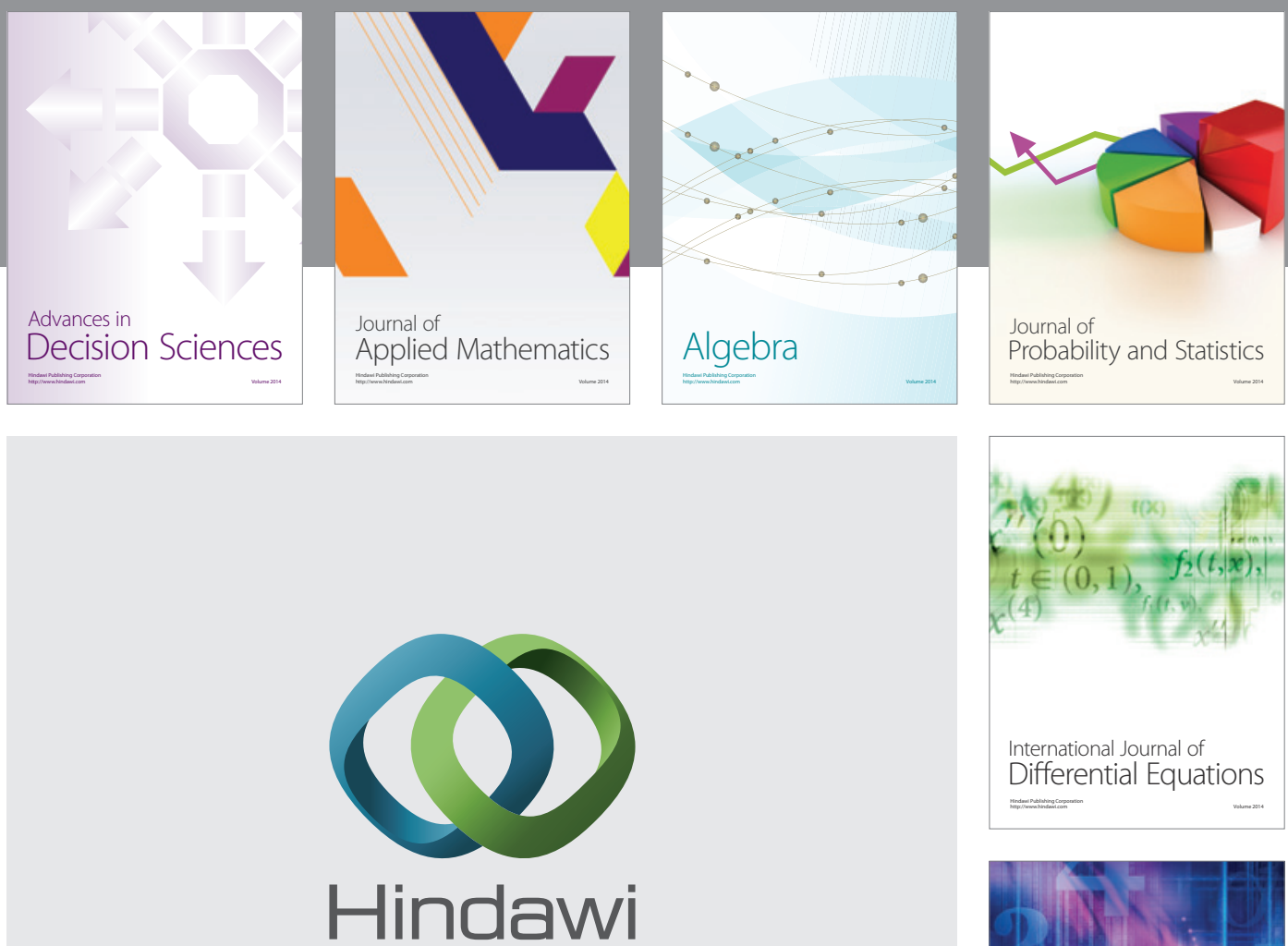

Submit your manuscripts at http://www.hindawi.com
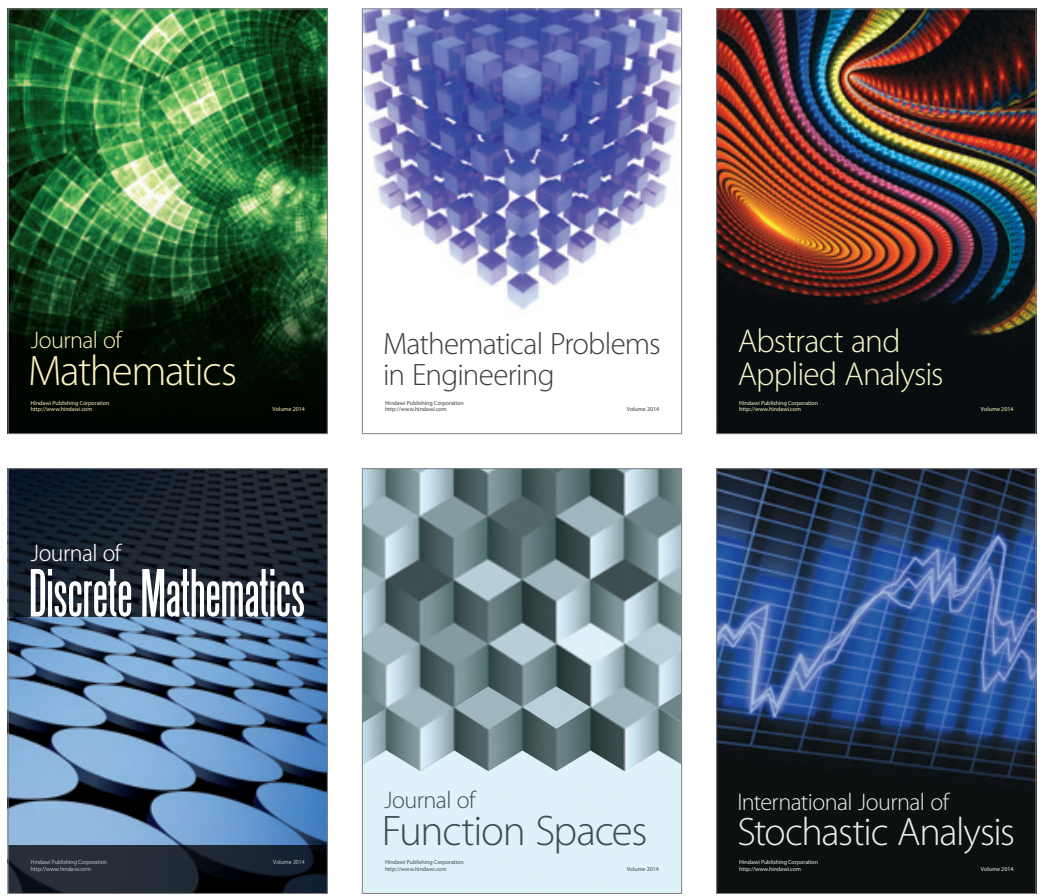

Journal of

Function Spaces

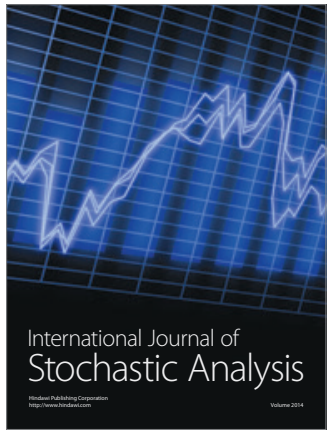

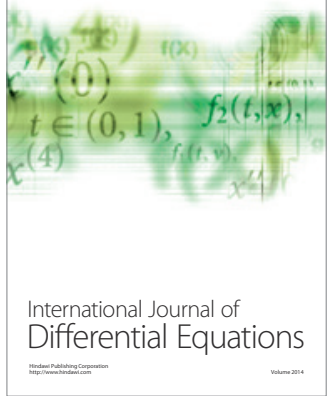
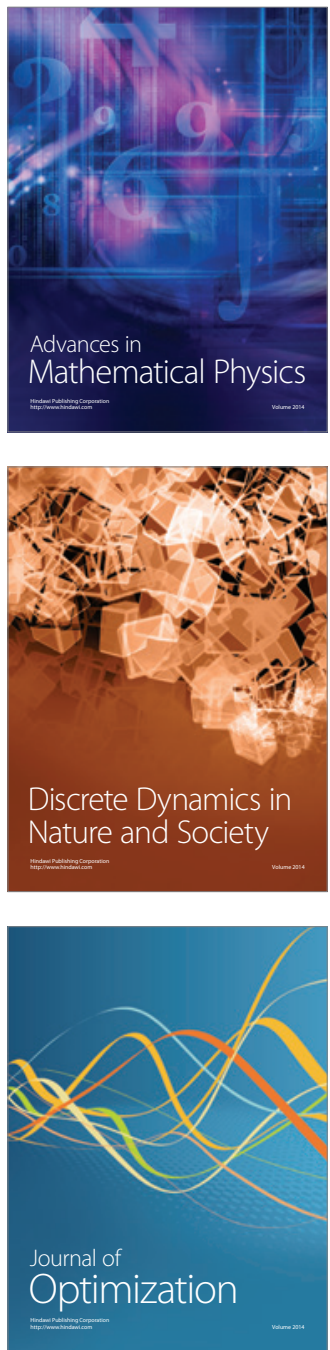